\title{
Current Approaches in Medical Technology for Effective Diagnosis
}

\author{
Rebecca Bello \\ Federal University, \\ Lokoja-Kogi State, Nigeria
}

\section{Doi:10.5901/ajis.2013.v2n12p23}

\begin{abstract}
Recent science and technology studies have analyse questions of expert and non-expert participating in science technology policy from an empirically grounded perspective, the issue offers a double contribution. It present a summary of the state of the act and an outline of the historical emergence of the participatory question, it distinguish four periods since the late nineteenth century each with a spec.ific relationship between expert and non-expert knowledge ranging from hybrid to politicized to an autonomous to a participatory relationship. In the early beginning of humankind, diseases were viewed with mysteries and were common occurrences that claimed many lives. Medical technology evolved slowly in response to high mortality rates associated with diseases example (malaria, yellow fever etc.) Advance medical technology has become the antidote for illnesses and diseases that formerly resulted in the destruction of entire societies. Advance medical technology now makes doctors and technologist to use mechanical techniques to diagnose patients. Medical technology slowly changed from the use of subjective evidence provided by patients to objective evidence obtained by mechanical and chemical technological devices. Patient's population now became concentrated in urban centres and large numbers of patient create economic incentives for doctors. This modern equipment saves time, energy and resources compared to the antediluvian approach. Therefore, multifarious diagnosis can be conducted with high degree of accuracy within a short period of time.
\end{abstract}

Keywords: Health care, medical intelligence, medical surveillance systems Nigeria, Nigerian health care system,

\section{1. introduction}

Objective, These study aim to review the state of medical technology in Nigeria and to provide recommendation on how to improve the technological system with regards to medical intelligence. To improve the knowledge of technologist, researchers and health personnel's. To imbibe the use of modern equipment in health care delivery, regulatory agencies, research institute and school laboratories.

Medical technology can be used to refer to the procedures, equipment, and processes by which medical care is delivered. Examples of changes in technology would include new medical and surgical procedures (e.g. angioplasty joint replacements), drugs (biological agents), and medical devices (CT scanners, implantable defibrillators) and new support systems e.g. electronic medical records and transmission of information.

Medical technology is the application of energy forms to diagnose and treat body systems using knowledge, procedures, and devices within the context of both science and technology in society. Medical technology cross disciplinary nature, medical technology applies energy forms to body systems. Energy forms are the physical forms of energy that exist in the universe, that is magnetic energy, thermal energy, electrical energy, chemical energy. Medical technology applies energy forms using devices such as x-ray machines, electrocardiographs, to diagnose and treat 
various than learning about energy forms and the body systems.

Medical technologist must also know about the appropriate procedures and devices for the diagnostic/therapeutic application of the energy forms to the body systems. Therefore, knowledge, procedures and devices simultaneously define the application of medical technology. Consequently, medical technology requires a foundation in both science and technology. In addition to science and technology, the basis for medical technology resides within the context of the social, political and cultural world.

\section{The History and Impact of Medical Technology}

In the early beginning of mankind, disease was viewed with mystery and was a common occurrence that claimed many lives. It was thought that disease was caused by negative interaction between environmental elements and body fluids. Poor health was considered a physical, social or personal deficiency within the patient lived. The four humours as described by Hippocrates (460377 B.C) and (131-201 A.D) were an attempt to qualitatively measure a deficiency or excess body fluids (blood, phlegm, non-medical terms for sputum, urine) with respect to the changing seasons, Hippocrates and Galen were the first documented western physicians to actively engage in rudimentary science of healing designed to increase the quality of life for their patients. Advance medical technology evolved slowly in response to the high mortality rates associated with diseases. Medical technology has become the antidote for illness and diseases that formerly resulted in the destruction of society.

\section{Current Approach to Medical Technology}

Medical technology is the application of devices, procedures and knowledge for diagnosing and treating of disease for the purpose of maintaining, promoting and restoring wellness while improving quality of life. These are the basis of medical technology.

Knowledge-: All technology starts with knowledge and this comes from multiple sources, this knowledge comes from the primary sources, educational institutions, governments and the collective or individual knowledge of those in the medical and science field.

Educational institutions-: Medical technology resides in hospital, clinic, universities, public libraries, medical laboratories and the internet. This knowledge consists of information or raw data. Specialized knowledge of medical technology is discipline based and usually resides in educational department of institution of higher learning. Specializations and unique disciplines of cardiology, neurology, homeopathic remedies, paediatrics anaesthesia and veterinary medicine.

Government-: A second source of medical technology knowledge comes from governments. There seems to be an increasing concern among democratic society for access to quality health care. The right to medical technology and services is beginning to be viewed as an obligation of the governments to it citizens by taking a serious interest in the application and administration of medical technology. In United States most of the medical technology research using new approve medical devices is been funded by government through universities and medical centres. Some of the common research and regulatory agencies include centres for disease control and prevention $(\mathrm{CDC})$, National Institutes of Health $(\mathrm{NIH})$, the Food and Drugs Administration and the office of health technology. These governmental agencies set agendas for research funding, investigate outbreak of infectious disease, monitor and approve new drugs and devices and evaluate medical technology.

Individual and collective knowledge-: The third source of medical technology knowledge is the individual and collective knowledge that reside within the mind of people and organizations that practice medicine and health care such as researchers, physicians, radiologist, laboratory workers, clinical insurance agencies, government and schools. Common knowledge about medical technology is both known and available, is characterize by it accessibility, availability and general 
understanding among the local population or society.

\section{Methods and Procedure}

Databases were searched for relevant literatures using the following keywords: Nigerian health care, Nigerian health care system, and Nigerian primary health care system. Additional keywords used in the search were as follows: United States (or Europe) health care dynamics, Medical Intelligence, Medical Intelligence systems, Public health surveillance systems, Nigerian medical intelligence, Nigerian surveillance systems, and Nigerian health information system. Literatures were searched in scientific databases PubMed and African Journals on line. Internet searches were based on Google and Search Nigeria.

\section{Equipments Use for the Effective Medical Laboratory Diagnosis}

Microscope, Stethoscope, Sphygmomanometer, Thermometer, Spectrophotometer, Autoclave, Centrifuge, Incubator, Distiller, Electrophoresis machine, Automatic tissue processor, Microtome machine, Medical X-ray machines, Water bath, Anaesthesia machine, Single Photon Emission Computing Tomography (SPECT) Scanning machine etc.

\section{Functions and Procedure of Operation of Some Selected Equipments}

Microscope is an instrument use for magnifying object that cannot be seen with the naked eyes, without microscope technologist, scientist, and clinical researchers will be lost. The microscope is an important part of a biology laboratory that help one observe specimen, microscope help magnify object 1000x their size. Microscope was invented by Antonio Phillip Van Leeuwenhoek (1632-1723). A Dutch trade man and scientist should be credited for the invention of this magical instrument, his crude microscope helped detect animalcules that is single cell organism, these led to research on bacteria, virus, fungi, protozoa as well as discovery of DNA. This proves that microscope are the most useful tools in the technology world, it is also useful in chemistry, metallurgy, and many other scientific field.

There are different types of microscope ranging from simple monocular to compound binocular, there is also electron microscope, metallurgical microscope, fluorescence microscope etc. Different kinds of microscope and their uses

Light microscope is also called the optical microscope. This is also a type of compound microscope that is used to view microorganisms. The microscope uses visible light and a system of lenses to magnify images of the manifold, light microscope can be simple which has single convex lens that is commonly observed in the loupe.

It can also be compound microscope use in school, college laboratory to observe plant and animal cell, bacteria, algae it uses two optical lenses called the ocular lens and the objective lens, these microscope are heavier and larger than the simple microscope. A separated set of lenses is use to focus the light into the eye to form image.

Metallurgical microscope is a microscope used to observed metal, plastic, ceramic as well as other material samples.

Electron Microscope is one of the most advanced microscope used today, the microscope is powered by a beam of electrons with very short wavelengths. These electron strike objects that come in its path and helps increase the resolution of the microscope. The electron microscope is use in the study cells like viral cell as well as larger molecules.

Autoclave is an instrument use for sterilization of materials use in the laboratory, surgical materials and biological waste, etc. The sterilization of instrument, food, glass vessels, medical liquid, culture medium etc. by saturated steam under pressure has been widely adopted by advance technology. 
Laboratory centrifuge is an instrument driven by a motor which spin liquid samples at high speed, by exerting a force greater than that of gravity. Which sediments particles (cells, bacteria, casts, parasites). The greater the outward pull due to rotation that is centrifugal force the more rapid and effective is the sedimentation, heavy particles sediment first followed by lighter particles. There is general purpose bench centrifuge which is design for clinical samples and sedimentation of cells, bacteria, parasites for microscopic examination e.g. blood, urine, cerebrospinal fluid, and micro haematocrit centrifuge use to diagnose and monitor anaemia. Although most modern centrifuge are fitted with an imbalance detector and lid interlock.

Stethoscope is an acoustic medical device for auscultation, or listening to the internal sound of an animal or human such as those in the heart and lungs. A simple stethoscope usually consists of a diaphragm or an open bell shaped structure which is applied to the body connected by rubber or plastic tubes to earpieces for the examiner.

Sphygmomanometer is an instrument for measuring blood pressure in the arteries. It consists of an inflatable cuff connected via a rubber tube to a column of mercury with a graduated scale. The cuff is applied to a limb usually the arm and inflated to exert pressure on a large artery until the blood flow stops. The pressure is then slowly released and with the aid of a stethoscope to listen to the pulse, it possible to feel the systolic and diastolic pressures which can be read on the scale.

\section{Result and Conclusion}

Medical intelligence and surveillance represent a very useful component in the health care system and control diseases outbreak, bio attack, etc. There is increasing role of automated-based medical intelligence and surveillance systems, in addition to the traditional manual pattern of document retrieval in advanced medical setting such as those in western and European countries.

Medical technology represent a very useful component in the health care system and even the educational system in the world, the provision of health care and quality education remain the functions of the government both federal, state and local level. The advancement of these equipment shows that technology is of utmost important in Nigeria and the world, there is increase in automated based medical equipment in the technology world in addition to manual in advance setting in the western and European countries.

In conclusion Nigeria should develop more by upgrading its facilities and training of medical personnel, technologist, and researchers, on the use of advance method of diagnosis and treatment for effective work.

Educational sectors need to improve it teaching aids in the institutions.

There should be adequate management with strong leadership principle.

\section{References}

David M. Cutter and McClellan (Sep-Oct 2001) is technological change in medicine worth it? FDA Guidance concerning demonstration of comparability of human biological products. (Review 2013) George B. Moseley, (2005) changing conditions for medical technology in health care industry. Henry J. Kaiser (2007) how changes in medical technology affect health care costs

Health Reform Foundation of Nigeria (HERFON) [Last accessed on 2010 Nov 23]. Available from: http://www.herfon.org/

Maternal Mortality in Nigeria. [Last accessed on 2010 Dec 16]. Available from: http://reproductiverights.org/en/feature/maternal-mortality-in-nigeria .

Nigeria National Health Conference 2009 Communique. Abuja, Nigeria. [Last accessed on 2010 Nov 5]. Available from: http://www.ngnhc.org .

Nnamuchi O. The right to health in Nigeria. 'Right to health in the Middle East' project, Law School, University of Aberdeen. Draft Report December 2007. [Last accessed on 2010 J une 21]. Available 
from: http://www.abdn.ac.uk/law/hhr.shtml .

Onwujekwe O, Onoka C, Uguru N, Nnenna T, Uzochukwu B, Eze S, et al. Preferences for benefit packages for community-based health insurance: An exploratory study in Nigeria. [Last accessed on 2010 June 21];BMC Health Services Research. 2010 10:162. Available from: http://www.biomedcentral.com/1472-6963/10/162 . [PMC free article] [PubMed] 
